\title{
Association between the TGFB1 -509C/T and TGFBR2 -875A/G polymorphisms and gastric cancer: A case-control study
}

\author{
LIXIA XU ${ }^{1}$, ZHIRONG ZENG ${ }^{1},{\text { BIN } \text { CHEN }^{1}, \text { XIAOQIN WU }}^{1}$, JUN YU $^{4}$, LING XUE $^{2}$, LINWEI TIAN $^{5}$, \\ YIMING WANG ${ }^{3}$, MINHU CHEN ${ }^{1}$, JOSEPH J.Y. SUNG ${ }^{4}$ and PINJIN HU ${ }^{1}$ \\ Departments of ${ }^{1}$ Gastroenterology, ${ }^{2}$ Pathology, The First Affiliated Hospital, and ${ }^{3}$ Zhongshan School of Medicine and \\ Center for Genome Research, Sun Yat-Sen University, Guangzhou; ${ }^{4}$ Institute of Digestive Disease and \\ Department of Medicine and Therapeutics, Li Ka Shing Institute of Health Sciences, and ${ }^{5}$ School of Public Health and \\ Primary Care, The Chinese University of Hong Kong, Hong Kong, SAR, P.R. China
}

Received October 8,2010; Accepted January 10, 2011

DOI: $10.3892 / \mathrm{ol} .2011 .249$

\begin{abstract}
The transforming growth factor- $\beta$ (TGF $\beta$ ) pathway plays an important role in various types of human cancer. However, the role of TGFB1 $-509 \mathrm{C} / \mathrm{T}$ and TGFBR2 $-875 \mathrm{~A} / \mathrm{G}$ polymorphisms in gastric cancer is controversial. We aimed to investigate the associations between these polymorphisms and gastric cancer susceptibility, clinicopathological parameters and survival. A case-control study was conducted in 1,010 gastric cancer patients and 1,500 healthy controls. Genotypes were determined by PCR-restriction fragment length polymorphism and DNA sequencing. Compared with the TT genotype, the TGFB1 $-509 \mathrm{C}$ allele $(\mathrm{CT} / \mathrm{CC})$ was significantly associated with a reduced risk of gastric cancer (OR, 0.71; 95\% CI, 0.58 $0.87 ; \mathrm{P}=0.001)$ and certain subtypes of gastric cancer including intestinal type (OR, $0.70 ; 95 \% \mathrm{CI}, 0.57-0.87 ; \mathrm{P}=0.001)$, poorly differentiated (OR, 0.67; 95\% CI, 0.54-0.85; $\mathrm{P}=0.001)$ and stage TNM III+IV (OR, 0.73; 95\% CI, 0.58-0.92; P=0.008). Compared with the TGFBR2 -875 GG genotype, carriers of the A allele (AA/AG) had a significantly decreased gastric cancer risk (OR, 0.58; 95\% CI, 0.62-0.91; $\mathrm{P}<0.001)$. A combination of the TGFB1 $-509 \mathrm{C}$ and TGFBR2 $-875 \mathrm{~A}$ alleles was associated with a further decreased risk of gastric cancer (OR, 0.42; 95\% CI, 0.32-0.57, P<0.001). No significant correlation was observed between polymorphisms and survival of gastric cancer patients. Our results suggest that both the TGFB1 -509 and TGFBR2 -875 polymorphisms contribute to a decreased gastric cancer risk. The TGFB1 -509 polymorphism affects certain subtypes of gastric cancer according to clinicopathological parameters. A combination of the TGFB1 $-509 \mathrm{C}$ and TGFBR2 -875 A alleles conferred a further decreased gastric cancer risk. These
\end{abstract}

Correspondence to: Professor Zhirong Zeng, Department of Gastroenterology, First Affiliated Hospital, Sun Yat-Sen University, Guangzhou 510080, P.R. China

E-mail: zengzhirong@vip.163.com

Key words: gastric cancer, polymorphism, clinicopathological characteristics, transforming growth factor- $\beta$ findings provide clues to the biological mechanisms that underline tumor heterogeneity.

\section{Introduction}

Gastric cancer is one of the most common malignancies worldwide and remains a leading cause of death in Asia (1). The association between gastric cancer and chronic inflammation has generally been recognized, and chronic Helicobacter pylori (H. pylori) infection has been found to be a significant risk factor for gastric cancer $(2,3)$. Only a small proportion of chronic $H$. pylori-infected individuals develop malignancies (2), suggesting that other factors, particularly host genetic factors, modulate the effects of inflammation in carcinogenesis.

The transforming growth factor- $\beta$ (TGF $\beta$ ) pathway plays an important role in inflammatory responses in the tumor microenvironment (4). TGF $\beta$ uniquely promotes the differentiation of human naive $\mathrm{CD}^{+} \mathrm{T}$ cells into $\mathrm{TH} 17$ cells, thereby becoming involved in the modulation of complicated inflammation pathways $(5,6)$. As a multifunctional cytokine, TGF $\beta 1$ regulates a number of important cellular responses, in addition to its role in cell differentiation, including apoptosis, cell migration, immune responses and angiogenesis. TGF $\beta 1$ initiates downstream signaling events by binding to the transforming growth factor $\beta$ receptor 2 (TGF $\beta$ R2), a constitutively active kinase which activates type 1 receptors by the phosphorylation of serine and threonine residues in the GS box and downstream signaling (7).

TGFB1 -509C/T (rs1800469) and TGFBR2 -875A/G polymorphisms (rs3087465) have been reported to be associated with gastric cancer risk (8-10). However, the results of studies from different geographical regions, ethnic groups and study groups are controversial (8-12). For example, certain studies found that carriers of TGFB1 -509T were associated with a decreased (8) or increased risk of gastric cancer (9). On the other hand, other studies have reported that the TGFB1 -509 $\mathrm{C} / \mathrm{T}$ polymorphism has no association with the risk of gastric cancer or patient survival $(11,12)$. The limitations of these studies should be considered when interpreting their findings. The majority of studies dismissed potential confounding 
factors such as age, gender, H. pylori status, tumor location and histological subtype, which may result in the bias and inaccuracy of results. In addition, it is known that TGFB1 and TGFBR 2 map to different chromosomes, i.e., 19q13.2 and $3 \mathrm{p} 24.1$, respectively, indicating that $T G F B 1$ and $T G F B R 2$ are independently inherited. Subsequently, variants of TGFB1 $-509 \mathrm{C} / \mathrm{T}$ and $T G F B R 2-875 \mathrm{~A} / \mathrm{G}$ exhibit a potential interaction with respect to gastric cancer risk.

This study aimed to investigate whether the TGFB1 $-509 \mathrm{C} / \mathrm{T}$ and $T G F B R 2-875 \mathrm{~A} / \mathrm{G}$ polymorphisms are correlated to the susceptibility of gastric cancer with respect to clinical and pathologic parameters. The combination of the genotypes of TGFB1 -509 and TGFBR2 -875 was also evaluated for their association with the risk of gastric cancer.

\section{Materials and methods}

Subjects. A population-based case-control study was conducted between 1999 and 2006 in Guangdong Province, China. The case series included 1,010 Chinese gastric cancer patients, newly diagnosed at the First Affiliated Hospital and Cancer Center of Sun Yat-Sen University, Guangzhou, China, from January 1999 to December 2006. The control series included 1,500 Cantonese healthy subjects from the general population of Guangzhou in southern China. Diagnosis was confirmed histologically as gastric cancer. The median duration of followup was 14 months (ranging from 0 to 98 months). Clinical data of the patients were collected from medical records and structured interviews were conducted using questionnaires. Patients diagnosed with gastric cancer received surgical resection of the tumors. Patients and control subjects provided written informed consent for participation in this study, and the study protocol was approved by the Clinical Research Ethics Committee of the Sun Yat-Sen University of Medical Sciences.

DNA extraction. Genomic DNA was extracted from paraffinembedded tissues of 1,010 gastric cancer patients using QIAamp DNA Mini kit (Qiagen, Hilden, Germany). Of these patients, 963 were included as the case group due to good DNA quality. Genomic DNA was also extracted from $2 \mathrm{ml}$ of peripheral blood of 1,500 healthy subjects using the Gentra Puregene Blood kit (Gentra Systems, Minneapolis, MN, USA). Of these, 787 subjects were selected as healthy controls frequency matched to the case group by age and gender. There were 655 males $(68 \%)$ and 308 females $(32 \%)$ in the gastric cancer case group, whereas the control group included 512 males (65\%) and 275 females (35\%). Frequency matching by age and gender was not complete, and thus these factors were further regulated in the multivariate data analysis stage. Extracted DNA was resuspended in UltraPure RNAse/ DNAse-free distilled water (Invitrogen, Hong Kong, China).

Genotyping analysis. TGFB1 -509C/T and TGFBR2 -875A/G genotyping was performed by polymerase chain reactionrestriction fragment length polymorphism (PCR-RFLP). Primer sequences for TGFB1 -509C/T and TGFBR2 -875A/G were: sense 5'-GAGACGCCTTGAAGTAACTG-3' and antisense 5'-AACCAAAGATGTTCTGAACTGA-3' for TGFBI -509C/T; and sense 5'-GCAAGAAAGGAAATTTGAAAGTT TGT-3' and anti-sense 5'-TCACCTGAATGCTTGTGTTTT-3' for $T G F B R 2-875 \mathrm{~A} / \mathrm{G}$. PCR was performed under the conditions: denaturation at $94^{\circ} \mathrm{C}$ for $10 \mathrm{~min}$, followed by $94^{\circ} \mathrm{C}$ (30 sec), $65^{\circ} \mathrm{C}(30 \mathrm{sec})$, and $72^{\circ} \mathrm{C}(30 \mathrm{sec})$ for 38 cycles, followed by a final extension at $72^{\circ} \mathrm{C}$ for $7 \mathrm{~min}$. PCR products were digested overnight at $37^{\circ} \mathrm{C}$ with $B s u 36 \mathrm{I}$ (New England BioLabs, Ipswich, MA, USA) for TGFB1 $-509 \mathrm{C} / \mathrm{T}$ and RsaI (New England BioLabs) for TGFBR2 $-875 \mathrm{~A} / \mathrm{G}$ genotypes, and then separated by electrophoresis on $3 \%$ agarose gel with ethidium bromide staining. PCR products were shown to be digested into 3 types of fragments (Fig. 1). To confirm the genotyping results, randomly selected PCR samples were examined by DNA sequencing (Fig. 2).

Statistical analysis. Hardy-Weinberg equilibrium in the controls was assessed using the $\chi^{2}$ test. The effects of the genotypes on the risk of gastric cancer were represented as odds ratios (OR) with $95 \%$ confidence interval (CI) by logistic regression. Relationships between the two polymorphisms and clinicopathological characteristics of the gastric cancer patients were compared using contingency tables and the Pearson's $\chi^{2}$ test. Since frequency matching was not complete, age and gender were included in all of the multiple logistic regression models. Kaplan-Meier survival curves and the log-rank test for trend were used to evaluate the relationship between the two polymorphisms and the prognosis from the date of primary diagnosis to the end of follow-up. The multivariate Cox regression analysis was performed to assess the prognostic value of the two polymorphisms with adjustment for age, gender, tumor size, grade, lymph node status and distant metastasis. Hazard ratios (HR) and 95\% confidence interval (CI) for each factor in the multivariate analysis were calculated from the Cox regression model. Differences were regarded as significant at $\mathrm{P}<0.05$. The above statistical analyses were performed using SPSS 13.0 software package (SPSS Inc, Chicago, IL, USA).

\section{Results}

Association of TGFB1 -509 C/T and TGFBR2 $-875 A / G$ polymorphisms with gastric cancer susceptibility. Among the controls, the genotype distribution for each SNP was in Hardy-Weinberg equilibrium $(\mathrm{P}>0.05)$. The frequencies of the polymorphisms in the cases and controls are shown in Table I. Compared with the TGFB1 -509 TT genotype, the CC and CT genotypes significantly decreased gastric cancer risk with odds ratios (ORs) of 0.70 (95\% CI, 0.53-0.94) and 0.72 (95\% CI, 0.58-0.89), respectively. Overall, the C carriers (CT or CC) were also associated with a lower gastric cancer risk when compared with the TT carriers (OR, 0.71; 95\% CI, 0.58-0.87; $\mathrm{P}=0.001$ ). Analysis of the TGFBR2 $-875 \mathrm{~A} / \mathrm{G}$ genotype polymorphisms showed a significant difference between the gastric cancer patients and controls $(\mathrm{P}<0.001)$. The AG genotype was associated with a lower gastric cancer risk when compared with the GG genotype (OR, 0.56; 95\% CI, 0.45-0.70; $\mathrm{P}<0.001$ ) (Table I).

Association of the TGFB1 -509C/T and TGFBR2 -875A/G polymorphisms with clinicopathological parameters. Stratified by the Lauren classification, the presence of the TGFB1 -509 C allele genotypes (CC or CT) was significantly associated with intestinal-type gastric cancer with an OR of $0.70(95 \%$ 

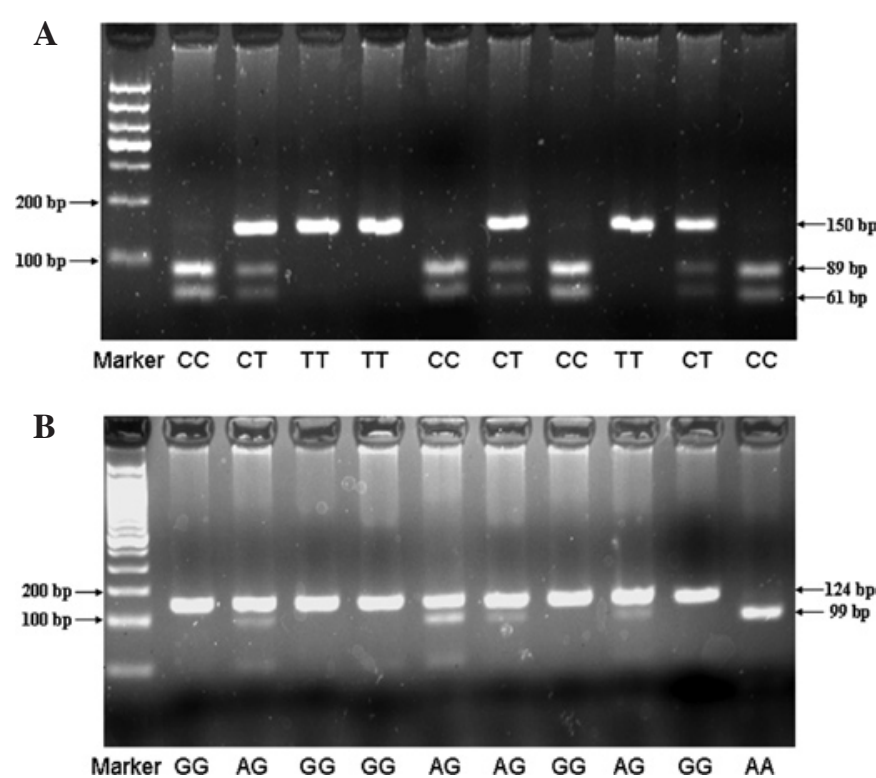

Figure 1. Genotyping of the TGFB1 $-509 \mathrm{C} / \mathrm{T}$ and TGFBR2 $-875 \mathrm{~A} / \mathrm{G}$ polymorphisms. (A) PCR-RFLP agarose gel electrophoresis of the TGFB1 -509 polymorphism showing the CC ( 89 and $61 \mathrm{bp}), \mathrm{CT}(150,89$ and $61 \mathrm{bp})$, and TT (150 bp) genotypes. (B) PCR-RFLP agarose gel electrophoresis of the TGFBR $2-875$ polymorphism showing the AA ( 99 and $25 \mathrm{bp}), \mathrm{AG}(124,99$ and $25 \mathrm{bp}$ ), and GG (124 bp) genotypes, while $25 \mathrm{bp}$ was too short to be detected.

CI, 0.57-0.87; $\mathrm{P}=0.001$ ), but not with diffuse- or mixed-type gastric cancer, as compared with the TT genotype. In contrast, the association between the TGFBR $2-875$ polymorphism and gastric cancer was not modified by the Lauren classification. Carriers of the AG genotype had a significantly decreased risk of intestinal-, diffuse- and mixed-type gastric cancer, compared to carriers of the GG genotype (Table II).

When the gastric cancers were stratified according to tumor differentiation, the effect of the TGFB1 -509 CC and CT genotypes remained significant on poorly differentiated gastric cancer with ORs of 0.63 (95\% CI, 0.45-0.88; $\mathrm{P}=0.006)$ and 0.69 (95\% CI, 0.54-0.88; $\mathrm{P}=0.002)$, respectively, but not on moderately or well-differentiated gastric cancer. However,
A

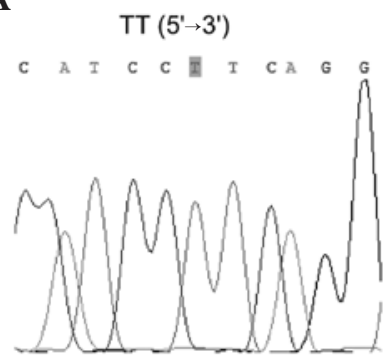

B
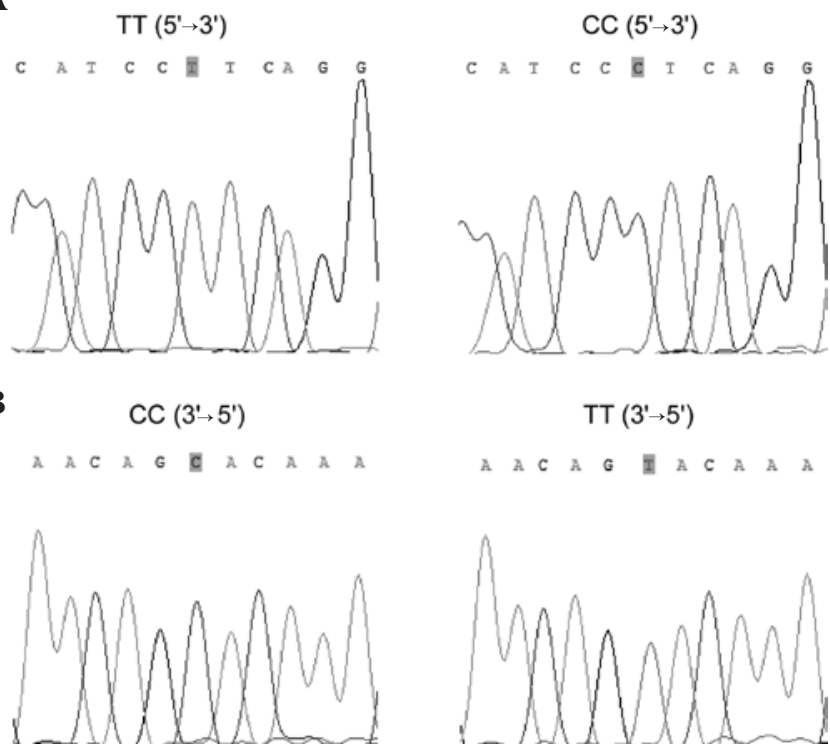

Figure 2. Sequencing analysis for genotypes of the TGFB1 $-509 \mathrm{C} / \mathrm{T}$ and $T G F B R 2-875 \mathrm{~A} / \mathrm{G}$ polymorphisms. (A) Sequencing analysis for genotypes of TGFB1 -509: TT and CC. (B) Reverse sequencing analysis for genotypes of TGFBR2 -875: GG and AA.

the association between the TGFBR2 -875 polymorphism and gastric cancer was not modified by tumor differentiation. The TGFBR2 -875AG genotype was found to be associated with both poorly differentiated and moderately or well-differentiated gastric cancer (Table III).

When the gastric cancer patients were divided into two subgroups according to TNM stage, the TGFB1 -509 CT genotype was found to be associated with TNM III/IV stage gastric cancer with an OR of 0.71 (95\% CI, 0.56-0.91; P=0.007), but not with TNM I/II stage gastric cancer, when compared with the TT genotype. In addition, the association between the TGFBR2 -875 polymorphism and gastric cancer was not modified by TNM stage. The TGFBR2 -875AG genotype was associated with both TNM I/II and TNM III/IV stage gastric cancer when compared to the GG genotype (Table IV).

Table I. Adjusted odds ratios (ORs) and 95\% confidence intervals (CIs) for gastric cancer in relation to the TGFB1 -509C/T and TGFBR2 -875A/G genotypes.

\begin{tabular}{|c|c|c|c|c|}
\hline Genotype & Control (\%) & Cases $(\%)$ & $\mathrm{OR}^{\mathrm{a}}(95 \% \mathrm{CI})$ & P-value \\
\hline \multicolumn{5}{|c|}{ TGFB1 -509C/T } \\
\hline $\mathrm{CT} / \mathrm{CC}$ & $536(69)$ & $578(61)$ & $0.71(0.58-0.87)$ & 0.001 \\
\hline $\mathrm{CC}$ & $133(17)$ & $142(15)$ & $0.70(0.53-0.94)$ & 0.016 \\
\hline $\mathrm{CT}$ & $403(52)$ & $436(46)$ & $0.72(0.58-0.89)$ & 0.002 \\
\hline TT & $245(31)$ & 367 (39) & 1 & \\
\hline \multicolumn{5}{|c|}{ TGFBR2 -875A/G } \\
\hline AG/AA & 305 (39) & $261(27)$ & $0.58(0.62-0.91)$ & $<0.001$ \\
\hline AA & 38 (5) & 39 (4) & $0.69(0.43-1.09)$ & 0.114 \\
\hline AG & $268(34)$ & $222(23)$ & $0.56(0.45-0.70)$ & $<0.001$ \\
\hline GG & $478(61)$ & $702(73)$ & 1 & \\
\hline
\end{tabular}

${ }^{a}$ Adjusted for age and gender. 
Table II. Adjusted odds ratios (ORs) and 95\% confidence intervals (CIs) for gastric cancer, stratified by Lauren classification, in relation to the TGFB1 $-509 \mathrm{C} / \mathrm{T}$ and TGFBR2 $-875 \mathrm{~A} / \mathrm{G}$ genotypes.

\begin{tabular}{|c|c|c|c|c|c|c|c|c|c|c|}
\hline \multirow[b]{2}{*}{ Genotype } & \multirow[b]{2}{*}{ Control } & \multicolumn{3}{|c|}{ Intestinal } & \multicolumn{3}{|c|}{ Diffuse } & \multicolumn{3}{|c|}{ Mixed } \\
\hline & & Case & $\mathrm{OR}^{\mathrm{a}}(95 \% \mathrm{CI})$ & $\mathrm{P}$-value & Case & $\mathrm{OR}^{\mathrm{a}}(95 \% \mathrm{CI})$ & P-value & Case & $\mathrm{OR}^{\mathrm{a}}(95 \% \mathrm{CI})$ & P-value \\
\hline \multicolumn{11}{|c|}{ TGFB1-509C/T } \\
\hline $\mathrm{CT} / \mathrm{CC}$ & 536 & 448 & $0.70(0.57-0.87)$ & 0.001 & 30 & $0.62(0.35-1.09)$ & 0.099 & 90 & $0.78(0.54-1.13)$ & 0.192 \\
\hline $\mathrm{CC}$ & 133 & 106 & $0.66(0.49-0.90)$ & 0.009 & 8 & $0.67(0.29-1.56)$ & 0.353 & 26 & $0.89(0.53-1.49)$ & 0.665 \\
\hline $\mathrm{CT}$ & 403 & 342 & $0.72(0.57-0.90)$ & 0.004 & 22 & $0.60(0.32-1.11)$ & 0.104 & 64 & $0.74(0.49-1.11)$ & 0.142 \\
\hline TT & 245 & 285 & 1 & & 22 & 1 & & 54 & 1 & \\
\hline \multicolumn{11}{|c|}{ TGFBR2 -875A/G } \\
\hline AG/AA & 305 & 203 & $0.58(0.46-0.72)$ & $<0.001$ & 15 & $0.64(0.34-1.19)$ & 0.158 & 36 & $0.53(0.35-0.79)$ & 0.002 \\
\hline $\mathrm{AA}$ & 38 & 26 & $0.59(0.35-0.99)$ & 0.049 & 5 & $1.81(0.67-4.93)$ & 0.244 & 6 & $0.61(0.23-1.58)$ & 0.304 \\
\hline $\mathrm{AG}$ & 267 & 177 & $0.57(0.46-0.72)$ & $<0.001$ & 10 & $0.48(0.24-0.99)$ & 0.047 & 30 & $0.51(0.33-0.79)$ & 0.003 \\
\hline GG & 478 & 552 & 1 & & 36 & 1 & & 105 & 1 & \\
\hline
\end{tabular}

adjusted for age and gender.

Table III. Adjusted odds ratios (ORs) and 95\% confidence intervals (CIs) for gastric cancer, stratified by tumor differentiation, in relation to the TGFB1 $-509 \mathrm{C} / \mathrm{T}$ and $T G F B R 2-875 \mathrm{~A} / \mathrm{G}$ genotypes.

\begin{tabular}{|c|c|c|c|c|c|c|c|}
\hline \multirow[b]{2}{*}{ Genotype } & \multirow[b]{2}{*}{ Control } & \multicolumn{3}{|c|}{ Poor } & \multicolumn{3}{|c|}{ Moderate or well-differentiated } \\
\hline & & Cases & $\mathrm{OR}^{\mathrm{a}}(95 \% \mathrm{CI})$ & P-value & Cases & $\mathrm{OR}^{\mathrm{a}}(95 \% \mathrm{CI})$ & P-value \\
\hline \multicolumn{8}{|c|}{ TGFB $1-509 \mathrm{C} / \mathrm{T}$} \\
\hline $\mathrm{CT} / \mathrm{CC}$ & 536 & 335 & $0.67(0.54-0.85)$ & 0.001 & 147 & $0.79(0.58-1.09)$ & 0.156 \\
\hline $\mathrm{CC}$ & 133 & 78 & $0.63(0.45-0.88)$ & 0.006 & 40 & $0.86(0.55-1.35)$ & 0.512 \\
\hline $\mathrm{CT}$ & 403 & 257 & $0.69(0.54-0.88)$ & 0.002 & 107 & $0.77(0.55-1.08)$ & 0.133 \\
\hline TT & 245 & 225 & 1 & & 83 & 1 & \\
\hline \multicolumn{8}{|c|}{ TGFBR2 -875A/G } \\
\hline AG/AA & 305 & 167 & $0.63(0.50-0.79)$ & $<0.001$ & 53 & $0.44(0.31-0.63)$ & $<0.001$ \\
\hline AA & 38 & 27 & $0.83(0.49-1.38)$ & 0.470 & 5 & $0.36(0.14-0.94)$ & 0.037 \\
\hline $\mathrm{AG}$ & 267 & 140 & $0.61(0.47-0.77)$ & $<0.001$ & 48 & $0.46(0.32-0.65)$ & $<0.001$ \\
\hline GG & 478 & 410 & 1 & & 180 & 1 & \\
\hline
\end{tabular}

${ }^{\mathrm{a} A d j u s t e d ~ f o r ~ a g e ~ a n d ~ g e n d e r . ~}$

Association of the TGFB1 $-509 C / T$ and TGFBR2 -875A/G polymorphisms with the survival of gastric cancer patients. Overall survival of the gastric cancer patients was analyzed using Kaplan-Meier survival curves for dependence on the TGFB1 -509C/T $(\mathrm{P}=0.859)$ and TGFBR2 $-875 \mathrm{~A} / \mathrm{G}$ genotypes $(\mathrm{P}=0.652)$, respectively. In the multivariate $\mathrm{Cox}$ regression analysis, the survival of gastric cancer patients was significantly associated with age $(\mathrm{P}=0.03)$, tumor staging $(\mathrm{P}=0.013)$, lymph node status $(\mathrm{P}<0.001)$ and distant metastasis $(\mathrm{P}<0.001)$. However, the TGFB1 $-509 \mathrm{C} / \mathrm{T}$ and TGFBR2 $-875 \mathrm{~A} / \mathrm{G}$ genotypes were not associated with outcome in gastric cancer patients (Table V).

Combination of the TGFB1 $-509 C / T$ and TGFBR2 $-875 A / G$ polymorphisms. We further investigated the effect of the combination of the TGFB1 $-509 \mathrm{C} / \mathrm{T}$ and TGFBR2 $-875 \mathrm{~A} / \mathrm{G}$ genotypes. As shown in Table VI, compared with the TGFBI -509 TT and TGFBR2 -875 GG genotypes, the combination of the TGFB1 -509CC/CT and TGFBR2 -875AA/AG genotypes was significantly associated with a decreased risk of gastric cancer (OR, 0.42; 95\% CI, 0.32-0.57; P<0.001) (Table VI).

Overall survival of the gastric cancer patients was analyzed using Kaplan-Meier survival curves. No statistical difference was noted between the variant combination of the TGFB1 -509 and TGFBR2 -875 genotypes $(\mathrm{P}=0.459)$. In the multivariate Cox regression analysis, age, tumor size, tumor grade, lymph node status and distant metastasis were significantly associated with the survival of gastric cancer patients. However, the combination of the TGFB1 -509 and TGFBR2 -875 genotypes was not associated with the outcome of the gastric cancer patients $(\mathrm{P}=0.766)$ (data not shown). 
Table IV. Adjusted odds ratios (ORs) and 95\% confidence intervals (CIs) for gastric cancer, stratified by TNM stage, in relation to the TGFB1 $-509 \mathrm{C} / \mathrm{T}$ and $T G F B R 2-875 \mathrm{~A} / \mathrm{G}$ genotypes.

\begin{tabular}{|c|c|c|c|c|c|c|c|}
\hline \multirow[b]{2}{*}{ Genotype } & \multirow[b]{2}{*}{ Control } & \multicolumn{3}{|c|}{ TNM I+II } & \multicolumn{3}{|c|}{ TNM III+IV } \\
\hline & & Cases & $\mathrm{OR}^{\mathrm{a}}(95 \% \mathrm{CI})$ & P-value & Cases & $\mathrm{OR}^{\mathrm{a}}(95 \% \mathrm{CI})$ & P-value \\
\hline \multicolumn{8}{|c|}{ TGFB1 -509C/T } \\
\hline $\mathrm{CT} / \mathrm{CC}$ & 536 & 143 & $0.83(0.61-1.15)$ & 0.260 & 339 & $0.73(0.58-0.92)$ & 0.008 \\
\hline $\mathrm{CC}$ & 133 & 30 & $0.70(0.44-1.13)$ & 0.146 & 91 & $0.78(0.56-1.08)$ & 0.138 \\
\hline $\mathrm{CT}$ & 403 & 113 & $0.88(0.63-1.22)$ & 0.436 & 248 & $0.71(0.56-0.91)$ & 0.007 \\
\hline TT & 245 & 77 & 1 & & 210 & 1 & \\
\hline \multicolumn{8}{|c|}{ TGFBR2 -875A/G } \\
\hline AG/AA & 305 & 64 & $0.59(0.43-0.82)$ & 0.002 & 141 & $0.54(0.43-0.69)$ & $<0.001$ \\
\hline AA & 38 & 7 & $0.53(0.23-1.22)$ & 0.137 & 21 & $0.66(0.38-1.14)$ & 0.134 \\
\hline $\mathrm{AG}$ & 267 & 57 & $0.60(0.43-0.84)$ & 0.003 & 120 & $0.53(0.41-0.68)$ & $<0.001$ \\
\hline GG & 478 & 61 & 1 & & 409 & 1 & \\
\hline
\end{tabular}

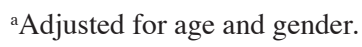

Table V. The Cox multivariate regression analysis of potential factors for overall survival in the gastric cancer patients.

\begin{tabular}{lrrr}
\hline Variable & HR $(95 \% \mathrm{CI})$ & P-value \\
\hline $\begin{array}{l}\text { Age } \\
\text { Gender } \\
\text { Female }\end{array}$ & 1.02 & $(1.00-1.03)$ & 0.030 \\
Male & 1 & & \\
& 1.12 & $(0.81-1.53)$ & 0.430
\end{tabular}

Tumor staging

\begin{tabular}{|c|c|c|c|}
\hline $\mathrm{T} 1$ & 1 & & \\
\hline $\mathrm{T} 2$ & 2.11 & $(0.40-10.16)$ & 0.350 \\
\hline $\mathrm{T} 3$ & 4.26 & $(0.995-18.26)$ & 0.050 \\
\hline $\mathrm{T} 4$ & 6.52 & $(1.49-28.50)$ & 0.013 \\
\hline \multicolumn{4}{|l|}{ Lymph node } \\
\hline Negative & 1 & & \\
\hline Positive & 2.60 & $(1.59-4.26)$ & $<0.001$ \\
\hline \multicolumn{4}{|l|}{ Metastasis } \\
\hline Negative & 1 & & \\
\hline Positive & 3.55 & $(2.50-5.02)$ & $<0.001$ \\
\hline \multicolumn{4}{|l|}{ Lauren classification } \\
\hline Intestinal & 1 & & \\
\hline Diffuse or mixed & 1.14 & $(0.71-1.82)$ & 0.600 \\
\hline \multicolumn{4}{|l|}{ Differentiation } \\
\hline Poor & 1 & & \\
\hline Moderate or well & 0.90 & $(0.62-1.31)$ & 0.590 \\
\hline \multicolumn{4}{|l|}{$T G F B 1-509 \mathrm{C} / \mathrm{T}$} \\
\hline TT & 1 & & \\
\hline $\mathrm{CT}$ & 0.93 & $(0.67-1.23)$ & 0.630 \\
\hline $\mathrm{CC}$ & 0.79 & $(0.47-1.31)$ & 0.360 \\
\hline \multicolumn{4}{|l|}{ TGFBR2 -875A/G } \\
\hline GG & 1 & & \\
\hline AG & 0.88 & $(0.39-1.98)$ & 0.750 \\
\hline $\mathrm{AA}$ & 0.87 & $(0.40-1.90)$ & 0.730 \\
\hline
\end{tabular}

\section{Discussion}

In the present study, we investigated the relationship between polymorphisms of the TGFB1 and TGFBR2 genes and the risk of gastric cancer in a low-risk Chinese population. TGFB1 -509 C (heterozygote or homozygote) and TGFBR2 AG genotypes were found to be associated with a reduced risk of gastric cancer, and this risk was particularly lower among subjects who carried the TGFB1 $-509 \mathrm{C}$ and TGFBR2 -875 A allele genotypes, suggesting that the TGFB1 $-509 \mathrm{C} / \mathrm{T}$ and $T G F B R 2$ $-875 \mathrm{~A} / \mathrm{G}$ polymorphisms were significant host protective biological factors that affected the risk of gastric carcinogenesis in southern China.

Previous studies on the association of the TGFB1 -509C/T polymorphism and gastric cancer are controversial. Li et al found that carriers of TGFB1 -509T were more susceptible to gastric cancer in northern China (9), which was consistent with our results (Table I). Jin et al reported that among a high-risk Chinese population in Nanjing, carriers of TGFB1 -509 T or TGFBR2 -875 A had a decreased risk of gastric cancer (8). On the other hand, no association was found between the TGFB1 $-509 \mathrm{C} / \mathrm{T}$ polymorphism and gastric cancer in a US population (12). Allele frequency patterns of the TGFB1 $-509 \mathrm{C} / \mathrm{T}$ polymorphism vary greatly between different geographical regions and ethnic groups. For example, the frequencies of the $-509 \mathrm{~T}$ allele among healthy controls were $0.468,0.461$ and 0.212 in Nanjing, Shandong and the US population, respectively $(8,9,12)$, while the $-509 \mathrm{~T}$ allele frequency in our healthy controls was 0.572 . As a result, the $-509 \mathrm{~T}$ allele acted as a major allele in our region, but as a minor allele in the above-mentioned regions. Ethnic differences in TGFB1 allele frequencies may be an explanation for the discrepancies in the findings between the present study and the study in the US population. However, findings of the latter study cannot explain the inconsistency in our study and that by Jin et al, where only minor differences in the $-509 \mathrm{~T}$ allele frequencies were noted in the control subjects. The discrepancy may be 
Table VI. Combined distribution of the TGFB1 $-509 \mathrm{C} / \mathrm{T}$ and TGFBR2 $-875 \mathrm{~A} / \mathrm{G}$ genotypes in the controls and gastric cancer patients.

\begin{tabular}{lcccr}
\hline Genotype $^{\mathrm{a}}$ & Control (\%) & Cases (\%) & OR $^{\mathrm{b}}(95 \% \mathrm{CI})$ & P-value \\
\hline 3 and 4 & $213(57)$ & $158(43)$ & $0.42(0.32-0.57)$ & $<0.001$ \\
1 and 2 & $153(37)$ & $266(63)$ & 1 & \\
\hline
\end{tabular}

a'1', the TGFB1 -509 TT genotype; '2', the TGFBR2 -875 GG genotype; '3', the TGFB1 -509CT/CC genotype and '4', the TGFBR2 -875 AG/AA genotype.

${ }^{\mathrm{b}}$ Adjusted for age and gender.

explained due to the different compositions of patients with various clinicopathological characteristics in the two studies. For the allele frequency of TGFBR2 -875A, similar frequencies were observed between Nanjing (0.197 in controls) and our population group ( 0.155 in controls) (8).

We further demonstrated that the TGFB1 $-509 \mathrm{C}$ allele was associated with a decreased risk of intestinal-type cancer. It has been widely accepted that intestinal- and diffuse-type gastric cancer differ with regards to age, gender and the process of carcinogenesis (13). The development of intestinal-type malignancy is a longer multistep process via gastric atrophy, intestinal metaplasia, dysplasia and ultimately intestinal-type carcinoma, which is initiated by $H$. pylori infection and by exposure to environmental toxins (14). $T G F B 1$, as a regulator of inflammation, may be involved in this multistep process. Messa et al reported that patients with $H$. pylori infection had elevated TGF $\beta 1$ levels in the gastric antrum, while TGF $\beta 1$ expression levels decreased following eradicating treatment (15). Kandulski et al reported that H. pylori-induced gastritis is associated with a recruitment of naturally occurring FOXP3(+) Treg cells that correlate with higher bacterial colonization and increased mucosal TGF $\beta 1$ expression, suggesting that $H$. pylori infection suppresses the maintenance and aggravation of gastric inflammation by activating the TGF $\beta 1$-FoxP-CD4 ${ }^{+} \mathrm{CD} 25^{\text {high }}$ Treg pathway (16). A similar phenomenon was observed in other animal studies $(17,18)$. Based on the above studies, we inferred that TGF $\beta 1$ plays an important role in the inhibition of $H$. pylori-induced gastritis. It has been reported that presence of the TGFBI $-509 \mathrm{C}$ allele is related to a higher production of TGF $\beta 1$ in the Chinese (19). Elevated TGF 1 may in turn affect the multistep process of gastric cancer initiated by $H$. pylori infection by activating $\mathrm{CD} 4{ }^{+} \mathrm{CD} 25^{\text {high }}$ Tregs, which contribute to a reduced incidence of intestinal-type gastric cancer.

This study showed that the TGFB1 $-509 \mathrm{C}$ allele was associated with poorly differentiated gastric cancer, but not with moderately or well-differentiated gastric cancer. Studies have shown that the p38 MAPK pathway is involved in the development of poorly differentiated gastric cancer (20-22). A specific p38 inhibitor was found to reduce the irregular dense membranous accumulation of F-actin in poorly differentiated gastric cancer cell lines (22). Moreover, activation of the phosphatidylinostiol 3-kinase (PI3K) via the p38 MAPK cascade caused the loss of cell-cell contact in poorly differentiated signet-ring cell carcinoma $(20,21)$. TGFB1 together with the IL1B gene are involved in a complex signal network. Therefore, the $T G F B 1$ polymorphism may be partially associated with poorly differentiated gastric cancer through inhibition of the p38 MAPK pathway.

In contrast, the TGFBR $2-875 \mathrm{~A} / \mathrm{G}$ polymorphism was found to be associated with decreased gastric cancer risk, and the association was not modified by pathological types, degrees of differentiation or TNM stages. A possible explanation for this phenomenon is that TGF $\beta$ suppresses carcinogenesis by suppressing tumorigenic inflammation and stroma-derived mitogens (23). In the TGF $\beta$ pathway, seven type I and five type II receptors paired in different combinations provide the receptor system for the entire TGF $\beta$ family. However, among the type II receptors, only TGF $\beta$ R 2 is able to bind to TGF $\beta$ to activate downstream signaling (23). Thus, TGF $\beta$ R2 acts as a key regulator in the TGF $\beta$ pathway. The TGFBR2 $-875 \mathrm{~A} / \mathrm{G}$ polymorphism may have an extensive effect in reducing the risk of gastric cancer by altering the protein expression of the $T G F B R 2$ gene to affect signal transduction.

Another noteworthy finding of this study was that $T G F B I$ $-509 \mathrm{C} / \mathrm{T}$ and TGFBR2 $-875 \mathrm{~A} / \mathrm{G}$ gene polymorphisms acted synergistically on the decreased risk of gastric cancer. The risk of gastric cancer was particularly lower among subjects who carried both the TGFB1 $-509 \mathrm{C}$ and TGFBR2 -875 A allele genotypes. TGFB1 and TGFBR2 map to different chromosomes but are essential components of the same signaling pathway. TGF $\beta$ signaling initiates carcinoma-immune cell cross-talk within the carcinoma cell and is involved in carcinogenesis, invasion and metastasis (24). TGF $\beta$ activation is induced by a number of mechanisms, including the expression of calpain and cathepsin D (24). Once activated, TGF $\beta$ binds to TGF $\beta$ R2 to activate downstream signaling (24). Thus, it is feasible that the combination of the TGFBI $-509 \mathrm{C} / \mathrm{T}$ and TGFBR $2-875 \mathrm{~A} / \mathrm{G}$ gene polymorphisms confer a synergistic effect on gastric cancer initiation and progression. This result confirmed the hypothesis that a combined assessment of functionally relevant common variants may aid in the characterization of cancer risk and prediction of disease aggressiveness (25).

Although studies have shown that TGF $\beta 1$ expression is closely linked to the prognosis of gastric cancer $(26,27)$, neither the polymorphisms of TGFB1 $-509 \mathrm{C} / \mathrm{T}$ and TGFBR2 $-875 \mathrm{~A} / \mathrm{G}$ nor the combination of the two SNPs revealed a correlation with patient survival in this study, consistent with the study in the US population (11). A possible explanation is that overall survival is determined, not only by factors involved in gastric cancer disease activity, but also by underlying diseases and other complications. Thus, SNPs alone may not be enough to affect the complex outcome. 
In conclusion, our study provides evidence that the TGFBI -509 CC, CT and TGFBR2 AG genotypes are significantly associated with a decreased risk of gastric carcinoma. The $T G F B 1-509$ C allele was found to be associated with a reduced risk of intestinal-type, poorly differentiated and TNM III/IV stage gastric cancer. Moreover, the combination of the TGFB1 $-509 \mathrm{C} / \mathrm{T}$ and $T G F B R 2-875 \mathrm{~A} / \mathrm{G}$ polymorphisms exhibited a synergistically decreased risk of gastric cancer in a southern Chinese population. These findings may provide clues to the biological mechanisms that underline tumor heterogeneity; thus, further studies are warranted.

\section{Acknowledgements}

This study was supported by the 'Doctoral Natural Science Foundation of Guangdong Province, China' (Grant no. 04300361) and 'Research Fund for the Doctoral Program of Higher Education of China' (Grant no. 20070558230).

\section{References}

1. Parkin DM, Bray F, Ferlay J and Pisani P: Global cancer statistics, 2002. CA Cancer J Clin 55: 74-108, 2005.

2. Moss SF and Blaser MJ: Mechanisms of disease: inflammation and the origins of cancer. Nat Clin Pract Oncol 2: 90-97, 2005.

3. Mantovani A, Allavena P, Sica A and Balkwill F: Cancer-related inflammation. Nature 454: 436-444, 2008.

4. Bierie B and Moses HL: Tumour microenvironment: TGFbeta: the molecular Jekyll and Hyde of cancer. Nat Rev Cancer 6: 506-520, 2006.

5. Yang L, Anderson DE, Baecher-Allan C, et al: IL-21 and TGF-beta are required for differentiation of human $\mathrm{T}(\mathrm{H}) 17$ cells. Nature 454: 350-352, 2008.

6. Veldhoen M, Hocking RJ, Atkins CJ, Locksley RM and Stockinger B: TGFbeta in the context of an inflammatory cytokine milieu supports de novo differentiation of IL-17producing T cells. Immunity 24: 179-189, 2006.

7. Massague J, Blain SW and Lo RS: TGFbeta signaling in growth control, cancer, and heritable disorders. Cell 103: 295-309, 2000.

8. Jin G, Wang L, Chen W, et al: Variant alleles of TGFB1 and TGFBR2 are associated with a decreased risk of gastric cancer in a Chinese population. Int J Cancer 120: 1330-1335, 2007.

9. Li T, Cao BW, Dai Y, Cui H, Yang HL and Xu CQ: Correlation of transforming growth factor beta-1 gene polymorphisms $\mathrm{C}-509 \mathrm{~T}$ and $\mathrm{T} 869 \mathrm{C}$ and the risk of gastric cancer in China. J Gastroenterol Hepatol 23: 638-642, 2008.

10. Zhang P, Di JZ, Zhu ZZ, et al: Association of transforming growth factor-beta 1 polymorphisms with genetic susceptibility to TNM stage I or II gastric cancer. Jpn J Clin Oncol 38: 861-866, 2008.

11. Guan X, Zhao H, Niu J, Tan D, Ajani JA and Wei Q: Polymorphisms of TGFB1 and VEGF genes and survival of patients with gastric cancer. J Exp Clin Cancer Res 28: 94, 2009.
12. Guan X, Zhao H, Niu J, Tang D, Ajani JA and Wei Q: The VEGF $-634 \mathrm{G}>\mathrm{C}$ promoter polymorphism is associated with risk of gastric cancer. BMC Gastroenterol 9: 77, 2009.

13. Yamaguchi $\mathrm{N}$ and Kakizoe T: Synergistic interaction between Helicobacter pylori gastritis and diet in gastric cancer. Lancet Oncol 2: 88-94, 2001.

14. Kountouras J, Zavos C, Chatzopoulos D and Katsinelos P: New aspects of Helicobacter pylori infection involvement in gastric oncogenesis. J Surg Res 146: 149-158, 2008.

15. Messa C, Di Leo A, Greco B, et al: Successful eradicating treatment of Helicobacter pylori in patients with chronic gastritis: gastric levels of cytokines, epidermal growth factor and polyamines before and after therapy. Immunopharmacol Immunotoxicol 18: 1-13, 1996.

16. Kandulski A, Wex T, Kuester D, et al: Naturally occurring regulatory $\mathrm{T}$ cells $\left(\mathrm{CD} 4^{+}, \mathrm{CD} 25^{\text {high }}, \mathrm{FOXP}^{+}\right)$in the antrum and cardia are associated with higher $H$. pylori colonization and increased gene expression of TGF-beta1. Helicobacter 13: 295-303, 2008.

17. $\mathrm{Rad} \mathrm{R}$, Brenner L, Bauer S, et al: CD $25^{+} / \mathrm{Foxp}^{+} \mathrm{T}$ cells regulate gastric inflammation and Helicobacter pylori colonization in vivo. Gastroenterology 131: 525-537, 2006.

18. Raghavan S, Fredriksson M, Svennerholm AM, Holmgren J and Suri-Payer E: Absence of $\mathrm{CD} 4{ }^{+} \mathrm{CD} 25^{+}$regulatory T cells is associated with a loss of regulation leading to increased pathology in Helicobacter pylori-infected mice. Clin Exp Immunol 132: 393-400, 2003.

19. Qi P, Chen YM, Wang $\mathrm{H}$, et al: $-509 \mathrm{C}>\mathrm{T}$ polymorphism in the TGF-betal gene promoter, impact on the hepatocellular carcinoma risk in Chinese patients with chronic hepatitis B virus infection. Cancer Immunol Immunother 58: 1433-1440, 2009.

20. Kobayashi M, Nagata S, Iwasaki T, et al: Dedifferentiation of adenocarcinomas by activation of phosphatidylinositol 3-kinase. Proc Natl Acad Sci USA 96: 4874-4879, 1999.

21. Xu Q, Karouji Y, Kobayashi M, Ihara S, Konishi H and Fukui Y: The PI 3-kinase-Rac-p38 MAP kinase pathway is involved in the formation of signet-ring cell carcinoma. Oncogene 22: 5537-5544, 2003

22. Atsumi T, Kato K, Uno K, et al: Pathophysiological role of the activation of p38 mitogen-activated protein kinases in poorly differentiated gastric cancer. Pathol Int 57: 635-644, 2007.

23. Massague J: TGFbeta in cancer. Cell 134: 215-230, 2008.

24. Bierie B and Moses HL: Transforming growth factor beta (TGF-beta) and inflammation in cancer. Cytokine Growth Factor Rev 21: 49-59, 2010

25. Kaklamani VG, Baddi L, Liu J, et al: Combined genetic assessment of transforming growth factor-beta signaling pathway variants may predict breast cancer risk. Cancer Res 65: 3454-3461, 2005

26. Nakamura M, Katano M, Kuwahara A, et al: Transforming growth factor betal (TGF-betal) is a preoperative prognostic indicator in advanced gastric carcinoma. $\mathrm{Br} \mathrm{J}$ Cancer 78: 1373-1378, 1998.

27. Vagenas K, Spyropoulos C, Gavala V and Tsamandas AC: TGFbeta1, TGFbeta2, and TGFbeta3 protein expression in gastric carcinomas: correlation with prognostic factors and patient survival. J Surg Res 139: 182-188, 2007. 